\title{
Development of a Polar Drive Shock Ignition Platform on the National Ignition Facility
}

\author{
L.J. Perkins, G P. Schurtz, R. Betti, R.S. Craxton, \\ K.N. LaFortune, A. Casner, A.V.Hamza, A.J. Comley, \\ X. Ribeyre, A.J. MacKinnon, P.W. McKenty, D.J. Strozzi, \\ D.T. Blackfield, T.Ma, D.S. Bailey, M.A. Lambert, S.Atzeni, \\ K.S. Anderson, R.C. Cook, G.V. Erbert
}

Revision 0

May 20, 2010 


\section{Disclaimer}

This document was prepared as an account of work sponsored by an agency of the United States government. Neither the United States government nor Lawrence Livermore National Security, LLC, nor any of their employees makes any warranty, expressed or implied, or assumes any legal liability or responsibility for the accuracy, completeness, or usefulness of any information, apparatus, product, or process disclosed, or represents that its use would not infringe privately owned rights. Reference herein to any specific commercial product, process, or service by trade name, trademark, manufacturer, or otherwise does not necessarily constitute or imply its endorsement, recommendation, or favoring by the United States government or Lawrence Livermore National Security, LLC. The views and opinions of authors expressed herein do not necessarily state or reflect those of the United States government or Lawrence Livermore National Security, LLC, and shall not be used for advertising or product endorsement purposes.

\section{Auspices Statement}

This work performed under the auspices of the U.S. Department of Energy by Lawrence Livermore National Laboratory under Contract DE-AC52-07NA27344. 


\title{
Development of a Polar Drive Shock Ignition Platform on the National Ignition Facility
}

\author{
L.J. Perkins ${ }^{1}$, G P. Schurtz ${ }^{2}$, R. Betti ${ }^{3}$, R.S. Craxton ${ }^{3}$, K.N. LaFortune ${ }^{1}$, A. Casner ${ }^{4}$, A.V.Hamza ${ }^{1}$, \\ A.J. Comley ${ }^{5}$, X. Ribeyre ${ }^{2}$, A.J. MacKinnon ${ }^{1}$, P.W. McKenty ${ }^{3}$, D.J. Strozzi ${ }^{1}$, D.T. Blackfield ${ }^{1}$, \\ T.Ma ${ }^{1}$, D.S. Bailey ${ }^{1}$, M.A. Lambert ${ }^{1}$, S.Atzeni ${ }^{5}$, K.S. Anderson ${ }^{3}$, R.C. Cook $^{1}$, G.V. Erbert ${ }^{1}$ \\ ${ }^{1}$ Lawrence Livermore National Laboratory, Livermore CA, USA \\ ${ }^{2}$ Centre Lasers Intenses et Applications, University of Bordeaux, France \\ ${ }^{3}$ Laboratory for Laser Energetics, University of Rochester, Rochester NY, USA \\ ${ }^{4} C E A, D A M, D I F$, Arpajon, France \\ ${ }^{5}$ Centre for Inertial Fusion Studies, Imperial College London, UK \\ ${ }^{5}$ Dipartimento di Energetica, University of Rome La Sapienza, Rome Italy
}

\section{Synopsis}

Shock ignition, a new concept for igniting thermonuclear fuel [1], offers the possibility for a near-term test of high-gain inertial confinement fusion on the National Ignition Facility at less than $1 \mathrm{MJ}$ drive energy [2] and without the need for new laser hardware [3]. In shock ignition, compressed fusion fuel is separately ignited by a strong late-time laser-driven shock and, because capsule implosion velocities are significantly lower than those required for conventional hotpot ignition, fusion energy gains of $\sim 60$ may be achievable on NIF at laser drive energies around $\sim 0.5 \mathrm{MJ}$, extending to $\sim 100$ at $1 \mathrm{MJ}$. Because of the simple all-DT target design, its in-flight robustness, the potential need for only 1D SSD beam smoothing, minimal early time LPI preheat, and use of present (indirect drive) laser hardware, this target should be easier to field on NIF than a conventional direct-drive hotspot ignition target [3]. A key immediate need is to determine the adequacy of low-mode drive uniformity and shock symmetry under NIF polar drive for the convergence ratios envisaged for these targets. Accordingly, we propose a set of phased experiments employing room temperature hydro-equivalent $\mathrm{CH}$ shells to (a) optimize NIF polar-drive symmetry and shock coupling under a combination of beam repointing, partial defocusing and phasing the time-dependent power balance from quad to quad, and (b) characterize the resulting laser-plasma interactions. The objective is to determine the optimum laser drive specifications that will then enable the fielding of a full cryogenic high-gain shock-ignition target on NIF ca. 2014.

\section{The Potential of Shock Ignition on NIF}

In contrast to conventional high-velocity hotspot ignition, the cryogenic shell in shock ignition is imploded at low velocity using laser drive of modest peak power and low total energy - Fig 1(a). The assembled fuel is then separately ignited from a central hotspot created by a strong, spherically convergent shock driven by the high intensity spike at the end of the laser pulse. Because the implosion velocity is significantly less than that required for hotspot ignition, considerably more fuel mass can be assembled for the same kinetic energy in the shell, potentially offering significantly higher fusion gains/yields for the same laser energy or, equivalently, retaining acceptable gains at appreciably lower drive energies. 
Fig 1(b) shows the results of 1D simulations for candidate NIF shock-ignited target designs with wetted-CH foam ablators [2] where the fusion energy gain curve is plotted as a function of the total delivered laser energy. The performance is also indicated for a near term, shock ignited candidate for NIF based on an all-DT fuel+ablator with the potential for gain 60 ( $\sim 30 \mathrm{MJ}$ yield) at around $\sim 0.5 \mathrm{MJ}$ drive energy [3]. Compared to conventional direct-drive, high velocity, hotspot ignition targets, this design is characterized by a stable, low-aspect-ratio thick shell that should be less susceptible to both initial speckle imprint and hot electron preheat.

(b)

(a)

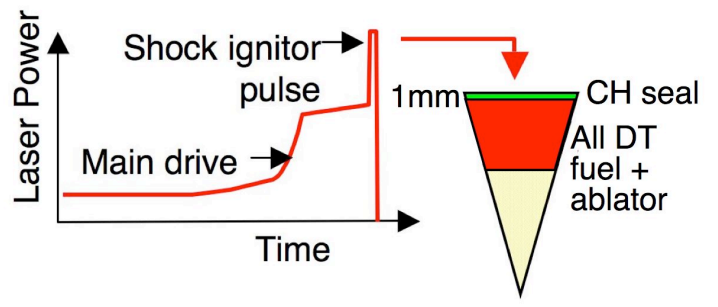

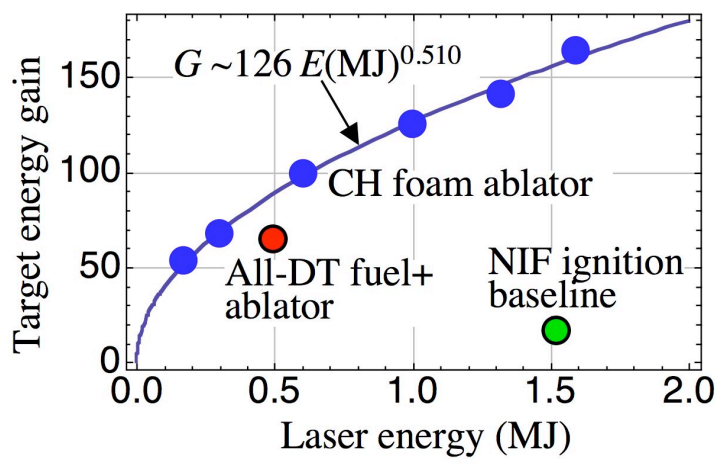

Fig 1. (a) Candidate all-DT NIF shock ignition target ( to scale) for nominal gain $\sim 60$ (30MJ yield) at a total drive energy of $\sim 0.5 M J$. (b) Energy gain curve (ID LASNEX) versus laser drive energy for NIF shock ignited targets (wetted foam ablators - blue points) together with corresponding performance for the all-DT fuel+ablator candidate design at $\sim 0.5 M J$ (red point)

Looking internationally, the HiPER [4] project requires a robust demonstration of ignition in an appropriate geometry in order to progress to construction. Shock ignition offers a highly attractive option. As such, the HiPER project will devote substantial effort to this experimental campaign if approved, including theoretical modeling, target design and experimental expertise. NIF is the only existing laser facility suited for this purpose.

\section{Prospects for a Near Term High Gain Experiment}

There is an opportunity to test a high-gain shock ignited target on NIF at $\sim 0.5 \mathrm{MJ}$ drive energy in the near-term ( $\sim 4$ years) with no new laser hardware required. This is due to its simple all-DT target design, its prospective in-flight robustness, the potential need for only 1D SSD beam smoothing and little or no early-time LPI preheat issues.

In Ref. 3, we have studied prospective routes for deploying shock ignition targets on NIF ranging from near term to longer term. The longer-term options have the potential for high fusion yields $(>100 \mathrm{MJ})$ using targets that are fully fusion-reactor-relevant, but at the expense of longer lead times due to requirements for new laser hardware. In contrast, the initial high gain target at $\sim 0.5 \mathrm{MJ}$ NIF drive energy (see Fig. 1 above) is considered a near-term prospect because it can be deployed with: (a) no modifications to laser hardware or signal path routing from the pre-amplifiers to the final optics - that is, no hardware modifications in the path: AWGs $\rightarrow$ PAMS $\rightarrow$ PABTS $\rightarrow$ main amplifiers $\rightarrow$ transport filters $\rightarrow$ conversion crystals $\rightarrow$ lenses $\rightarrow$ phase plates, and (b) no disruption to the baseline indirect drive ignition campaign. 
Shock-ignited targets in the next few years would be fielded on NIF under polar drive as proposed for standard NIF direct drive targets [5-7] but can employ day-1 indirect drive phase plates [8]. The key immediate need for shock ignition on NIF is to verify the adequacy of low-mode drive uniformity and shock symmetry under polar drive for the convergence ratios predicted for these shock ignited targets. Accordingly the primary objective of the preparatory experiments in this facility time proposal is to determine the optimum laser specifications - i.e., pulse shape, pointings, focusings and power phasing per quad - that will then enable the fielding of a full cryogenic high-gain shock ignition target $\geq 2014$. Another important objective is to identify whether there are any significant laser-plasma interactions at relevant laser intensities and whether they are detrimental or beneficial to the performance of the target [3].

\section{NIF Beam Geometry - Optimization of Polar Drive Symmetry}

Under polar drive (PD), the NIF beams are retained in the present indirect drive port configurations but adequate direct drive uniformity is potentially achievable by a combination of repointing and partial defocusing of laser beams and phasing the independently-programmable power balance from quad to quad. A PD experimental platform has been developed as part of the National Ignition Campaign for commissioning nuclear diagnostics $[8,9]$. Successful PDD shots performed on NIF during 2009 were consistent with 2-D simulations. Design studies are now in progress with our hydrodynamic implosion codes to determine the initial laser drive specifications that can then be experimentally tested under the shot schedule in this proposal.

A promising initial configuration has been identified wherein half the NIF quads (96 "main beams") are given the compression pulse and optionally some of the shock pulse, and the other half ( 96 "ignitor beams") are given just the shock portion of the pulse (see Fig. 2). Separate focusing and pointing parameters are used for the main and ignitor beams. The ignitor beams are launched at late time when the capsule has converged a factor of $\sim$ three from its original diameter, and are focused at the converged capsule radius at that time. The main beams are focused on the initial radius, optimized to provide a uniform compression. This overall configuration provides a "zooming" that should enhance the shock-drive intensity and provide enhanced coupling of the shock pressure pulse to the compressed core. The high-power portion of the main beams, while not optimally focused, would still contribute to the shock drive.

An important feature of the proposed configuration is that each ring of quads is split into quads with alternating pulse shapes (compression and shock), as illustrated in Fig. 2. This is necessary, as all four beams in each quad are required to have the same time-dependent pulse shape. (At a later stage it is possible to remove this constraint by reconfiguring signal routings in the pre-amplifier modules, e.g., to provide the compression pulse shape to two beams in each quad.) In order to provide azimuthal symmetry, the beams are all repointed in the horizontal direction so that, for example, the inner ring of quads at $23.5^{\circ}$ still produces fourfold symmetry in the azimuthal direction. Thus, each quad is split into four independently pointed/focused beams, resulting in a total of 16 rings of beams, 8 top and 8 bottom. With the additional possibility of separately tuning the pulse shapes of each quad, a large parameter space is available for the optimization of symmetry. Wherever possible, designs are preferred in which the two rings of each quad are given the same repointing (i.e., pointed to the dashed circle in Fig. 2) to reduce the effects of power imbalances within a quad. The additional possibility of 
separately tuning the pulse shapes of each quad offers a large parameter space for optimization of symmetry.

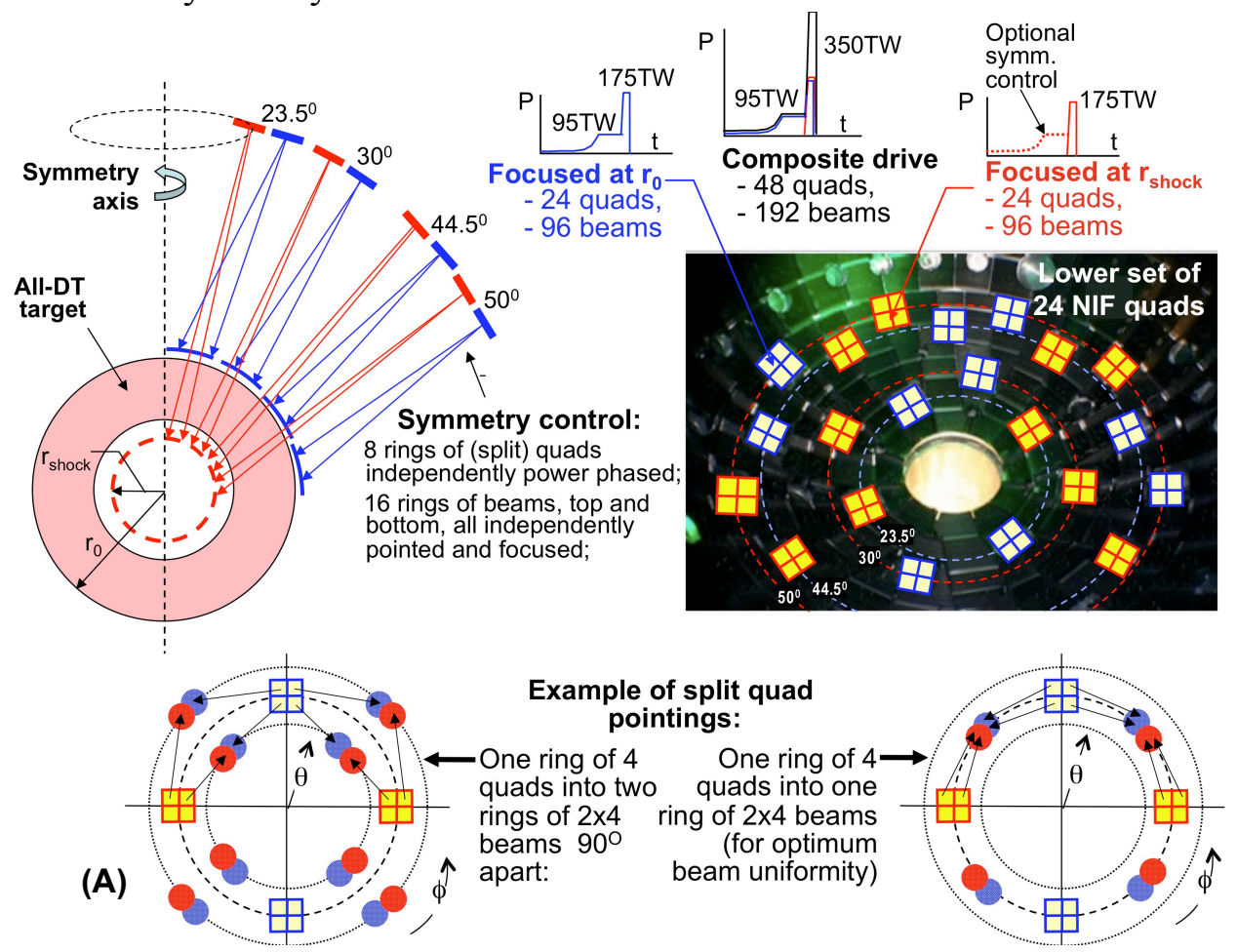

(B)

Fig 2. Proposed initial "zooming" configurations for optimizing polar drive symmetry. Half the quads (96 "main" beams) are focused at the capsule radius at time zero while the other 96 ("ignitor") beams are focused around the reduced critical radius at time of shock launch. Split quad pointing and focusing are employed to optimize beam uniformity in both polar $(\theta)$ and azimuthal $(\phi)$ angles. A total of up to 16 rings with independent pointing and focusing, 8 top and 8 bottom, can be used for each set of beams. Option $B$ provides only 4 rings of (split) quads and 8 rings of beams, top and bottom, but with better compensation for any beam-to-beam nonuniformity within a quad

Using this configuration, initial optimizations with the two-dimensional hydrodynamics code $S A G E$ carried out in a similar way to Ref. 8 have identified parameters for the compression beams that provide good uniformity for the initial phase of the compression, for an all-DT design. The parameters include two repointings (vertical and horizontal) and a defocus distance for each ring of beams. These parameters will be adjusted as the 1D and 2D designs evolve.

In terms of NIF laser power requirements, the main drive pulse for fuel assembly will be at a modest peak power $P_{\text {main }} \sim 50-90 \mathrm{TW}$ while the shock pulse $P_{\text {shock }}$ will require higher peak powers of $\sim 250-350 \mathrm{TW}$, for a total drive energy of $\sim 0.5-0.7 \mathrm{MJ}$. For comparison, the baseline NIF indirect-drive ignition target with a $\mathrm{CH}$ ablator requires equivalent peak powers/beam of $\sim 420 \mathrm{TW}$ at $\sim 1.6 \mathrm{MJ}$ drive energy. These are relative to maximum NIF peak powers at $3 \omega$ of $\sim 450-500 \mathrm{TW}$ depending on scenario. We have performed initial validations of our preliminary shock ignition pulse shapes with the NIF Laser Performance Operations Model (LPOM) [10]; results indicate that temporal contrasts should be achievable in the main amplifiers and that proposed pulse shapes do 
not pose any equipment protection issues. The shock launch time $t_{\text {shock }}$ determines the arrival of the shock ignition pulse relative to the hydro bounce of the stagnating fuel. For the targets envisaged in this proposal, ignition shock synching requires a shock pulse launch window - that is, the permissible spread of $t_{\text {shock }}-$ of $\sim 0.4 \mathrm{~ns}$ with a $10-90 \%$ risetime requirement of $\leq 0.35 \mathrm{~ns}$. An initial simulation of the pulse shape to be supplied by the NIF AWGs - that is, the pulse shape required as input to LPOM - indicates that the fast risetime can be delivered with no hardware changes in the front end [3].

\section{Proposed Experimental Plans and Objectives}

Our proposed plan for FY 2011-2013 is to field three phases of experiments as shown in Table 1. Diagnostic requirements are summarized in the accompanying NIF PowerPoint template and consist of the primary diagnostics of the NIF nuclear diagnostic suite (Neutrons Time of Flight detectors, Magnetic Recoil Spectrometer, WRF proton spectrometer, etc.) and gated X-ray imagers (or Image Plates), operational from the NIC campaign. Minor changes to the FFLEX and backscattering light diagnostics (FABS, NBI) will be requested in Phase 1.

Table 1. Shock ignition on NIF - Proposed experimental plan (Phases 1-3) to optimize polar drive symmetry and shock coupling together and characterize associated laserplasma interactions.

\begin{tabular}{|c|c|c|c|c|}
\hline & $\begin{array}{c}\text { Phase } 1 \text { - } 2011 \\
8 \text { shots } \\
\end{array}$ & $\begin{array}{c}\text { Phase } 2 \text { - } 2012 \\
10 \text { shots } \\
\end{array}$ & $\begin{array}{c}\text { Phase } 3-2013 \\
10 \text { shots } \\
\end{array}$ & $\begin{array}{c}\text { Phase } 4 \text { - } \geq 2014 \\
\text { Shots TBD }\end{array}$ \\
\hline Experiments & $\begin{array}{l}\text { PD Symmetry: } \\
\text { (i) Low CR (ii) High CR. } \\
\text { Characterization of TPD }\end{array}$ & $\begin{array}{l}\text { Integrated implosions } \\
\text { w/shock. Optimization of } \\
\text { laser geometries and shock } \\
\text { timing. Characterization of } \\
\text { late time SRS SBS }\end{array}$ & $\begin{array}{l}\text { Integrated implosions } \\
\text { w/shock. Final tunings. }\end{array}$ & $\begin{array}{l}\text { Cryogenic shock } \\
\text { ignition }\end{array}$ \\
\hline Targets & Room-temp $\mathrm{CH}$ shells & Room-temp CH shells & Room-temp CH shells & $\begin{array}{l}\text { (i) Cryo THD, (ii)Cryo } \\
\text { DT }\end{array}$ \\
\hline Fill gases & $\mathrm{Ar}, \mathrm{D}-{ }^{3} \mathrm{He}$ & $\mathrm{Ar}, \mathrm{D}_{2}, \mathrm{D}-{ }^{3} \mathrm{He}$ & $\mathrm{Ar}, \mathrm{D}_{2}, \mathrm{D}-{ }^{3} \mathrm{He}$ & N.A \\
\hline $\begin{array}{l}\text { Laser Energy } \\
\text { Requirements }\end{array}$ & $250-500 \mathrm{~kJ}$ (compr.) & $\begin{array}{l}250-500 \mathrm{~kJ} \text { (compr.) } \\
250-300 \mathrm{~kJ} \text { (shock) }\end{array}$ & $\begin{array}{l}250-500 k J \text { (compr.) } \\
250-300 k J \text { (shock) }\end{array}$ & TBD \\
\hline $\begin{array}{l}\text { NIF Power } \\
\text { Requirements }\end{array}$ & 50-90TW (compr.) & $\begin{array}{l}\text { 50-90TW (compr.) } \\
\text { 200-350TW (shock) }\end{array}$ & $\begin{array}{l}\text { 50-90TW (compr.) } \\
\text { 200-350TW (shock) }\end{array}$ & TBD \\
\hline
\end{tabular}

Other requirements: Phase plates - day-1 (indirect drive) partially defocussed. Beam smoothing - 1D SSD ( $\rightarrow$ Multi-FM 1D SSD when available). Bandwidth $-0.2 \mathrm{THz}(\rightarrow 0.5$ when available). Pulse lengths $-\sim 15 \mathrm{~ns}$ (compression) $\sim 0.8 \mathrm{~ns}$ (shock),; shock pulse risetime $\leq 0.35 \mathrm{~ns}$

The objective of Phases 1-3 is to obtain optimized drive geometries and power pulse shapes together with an assessment of LPI and electron transport issues that will then enable the fielding of full cryogenic shock-ignition targets in Phase 4, ca. 2014. This latter campaign (to be defined) aims at reaching the Ignition Threshold Factor required in cryo-THD [11] leading to full shock ignition attempts in cryo-DT at high gain, that is $\sim 30 \mathrm{MJ}$ at $0.5 \mathrm{MJ}$ drive energy [3]. We will also benefit from on-going [12] and future NIF-PD-like shock ignition preparatory experiments on the OMEGA laser facility [13].

The first set of implosion shots in Phase 1 will be devoted to the validation and initial tuning of PD configurations suitable for the compression phase of shock ignition. 
They will employ surrogate room-temperature $\mathrm{CH}$ targets that are hydro-equivalent to the cryo targets envisaged for Phase 4. Main observables will be the shape of the imploding shell, the shape of the compressed core and the areal density. The success of this first phase is a condition for integrated implosion experiments in 2012- 2013 in Phases 2-3 using both compression and shock pulses.

The initial diagnostic for initial PD symmetry measurements will be gated x-ray backlighting of the imploding shell. Given that only half the beams are required for target compression in Phase 1 (see Fig. 2) there are ample beams remaining for backlighting. This technique has been demonstrated on OMEGA [14]. Additionally, capsules will include argon in the fill, enabling gated x-ray images to be obtained in Ar self-emission from the core of the imploded capsules. These images will provide a diagnostic of the symmetry of the compressed core.

Gated x-ray detectors (GXD) will be positioned along two orthogonal lines of sight defined by the polar and equatorial DIMs, where the GXD in each DIM consists of a filtered x-ray pinhole snout mounted in front of a microchannel plate (MCP) detector. Each MCP detector has four strip lines, which are independently gated in time. The strip timings can be referenced to the start of the laser drive via the NIF cross-timing system in order to determine both the drive on the $\mathrm{CH}$ shell and the time of peak $\mathrm{x}$-ray brightness (x-ray bang time), in addition to recording the shape of the imploded core.

Phase 1 will require the tuning of the power balance among cones, and possibly, the investigation of two different PD options. Five to eight shots will therefore be necessary. Neutron imaging could be fielded by CEA to complement the x-ray imaging of the core and can be deployed on at least 2 of the 10 shots. The shell areal density will be inferred from the spectrum of protons arising from $\mathrm{D}^{3}{ }^{3} \mathrm{He}$ secondary fusion reactions. Thus, at least two shots including a $\mathrm{D}_{-}^{3} \mathrm{He}$ mixture will also be fielded for comparison.

In order to gain insight into the role of the two-plasmon decay (TPD) instability as a possible source of hot $(\sim 70 \mathrm{keV})$ electrons during the compression phase [15], we proposed to record and correlate its optical signature with the hard x-ray spectrum resulting from the interaction, an approach demonstrated at OMEGA [16]. The x-ray spectrum will be recorded with the filter fluorescer array FFLEX. This currently consists of eight time-integrated channels (seven channels defined by fluorescence cross-section edges in the range $20-80 \mathrm{keV}$, and one broadband channel centred on $150 \mathrm{keV}$ ), and two time-resolved channels detecting $\mathrm{x}$-rays in the range $>250 \mathrm{keV}$ and $>450 \mathrm{keV}$ with an $800 \mathrm{ps}$ time resolution. Time-resolved hard $\mathrm{x}$-ray detectors can discriminate between emission resulting from LPI-generated hot electron production and emission generated at the time of peak compression, in addition to providing a time record that can be compared to the corresponding time history of optical LPI signatures. Based on this, it may be desirable to make a small change to the standard FFLEX configuration such the time-resolved channels 9 and 10 are reconfigured to look at energy ranges $<100 \mathrm{keV}$. This would involve changing the filtering combinations currently employed in those channels.

The optical signature of the TPD instability consists of half-harmonic scattered radiation, such as $3 / 2 \omega(234 \mathrm{~nm})$ light generated via Thomson up-scattering of a laser photon off a TPD plasmon. However the NIF optical diagnostics, namely the full aperture backscatter system (FABS) and near-backscatter imaging (NBI) system, are currently configured to record light in the SRS (450-750nm) and SBS (approximately 348-352nm) spectral bands, which are of prime interest to the indirect drive campaign. In order to record the $3 / 2 \omega$ TPD signature, it is necessary to make a change to the standard 
configuration of the optical diagnostics. A number of options are currently being considered.

Phase 2 will also employ $\mathrm{CH}$ shells with $\mathrm{D}_{2}$ fill gases where we expect to observe a significant enhancement of neutron yield from deuterium shocked targets, as has been recently observed on OMEGA [12]. We will also explore the use of $\mathrm{D}^{3} \mathrm{He}$ target fills to permit assessment of the areal density and the stability associated with the initial level of beam smoothing through charged particle diagnostics and yield measurements. Neutron number and spectrum will be the major indicators for proper shock synchronicity and efficiency. Core imaging will be used to diagnose shock symmetry. A total of $\sim 10$ shots will be required to determine the best shock timing. Raman backscattered light during the shock pulse and the hot electron spectrum (diagnosed with FFLEX) are crucial measurements that relate to the efficiency of shock ignition.

Phase 3 will use similar targets and laser pulses, which will probably include minor amendments incorporating lessons learned from Phase 2 and improvements to the 1D design. These amendments, which could include changes to the laser pulse shapes and the implosion velocity, would require fine tuning of the polar drive parameters. The same diagnostics would be used as in Phase 2.

The experimental campaigns for Phase 4 - i.e., fielding of full cryo THD and DT ignition targets - will be defined at a future stage as the definitive results become available from Phases 1-3

\section{References}

[1] R. Betti , C.D. Zhou , K.S. Anderson , L.J Perkins, A.A. Solodov, "Shock Ignition of Thermonuclear Fuel with High Areal Density", Phys. Rev. Lett., 98, 155001 (2007)

[2] L.J. Perkins, R.Betti, K.N. LaFortune, W.R., Williams, "Shock Ignition: A New Approach to High Gain Inertial Confinement Fusion on the National Ignition Facility", Phys. Rev. Lett., 103, 045004 (2009)

[3] L.J.Perkins, R.Betti, G.Schurtz, R.S.Craxton, A.M.Dunne, K.LaFortune, A.Schmitt, P.McKenty, D.Bailey, M.Lambert, X.Ribeyre, W.Theobald, D.Strozzi, ,D.Harding, A.Casner, S.Atzeni, G.Erbert, K.Andersen, M.Murakami, A.Comley, R.Cook, R.Stephens, "On the Fielding of a High Gain Shock Ignited Target on the National Ignition Facility in the Near Term", Lawrence Livermore National Laboratory Technical Report, LLNL-TR-428513, April 2010

[4] A. M. Dunne, "A High Power Laser Fusion Facility for Europe”, Nature Physics 2 2 (2006)

[5] S. Skupsky, J. A. Marozas, R. S. Craxton, R. Betti, et al., "Polar direct drive on the National Ignition Facility", Phys. Plasmas 11, 2763 (2004)

[6] J. A. Marozas, F. J. Marshall, R. S. Craxton, I. V. Igumenshchev, , et al.,, "Polardirect-drive Simulations and Experiments" Phys. Plasmas 13, 056311 (2006)

[7] P.W. McKenty, et al., "Mulitidimensional Numerical Investigation of NIF Polar Drive Designs with Full Beam Smoothing", Inertial Fusion Sciences and Applications (IFSA) Conference, Kobe, Japan (2007) 
[8] A.M. Cok, R. S. Craxton, P.W. McKenty, "Polar-drive Designs for Optimizing Neutron Yields on the National Ignition Facility", Phys. Plasmas 15082705 (2008)

[9] P. McKenty et al., "Design of High-Neutron-Yield, Polar-Drive Targets for Diagnostic Activation Experiments on the NIF", 6th IFSA, San-Francisco (2009).

[10] M. Shaw, W. Williams, R. House, C. Haynam, "Laser performance operations [11] B. K. Spears et al. "Predicting ignition implosion performance using lowdeuterium surrogates". 6th IFSA, San Francisco (2009). LLNL-PRES-416367

[12] W. Theobald, R. Betti, V.A. Smalyuk, K.S. Anderson, et al, "Shock Ignition Experiments on OMEGA at NIF Relevant Intensities, Phys. Plasmas. to be published (2010)

[13] R. Betti et al., FY11 OMEGA Laboratory Basic Science Proposal.

[14] R. S. Craxton, F. J. Marshall, M. J. Bonino, R. Epstein, et al., "Polar direct drive: Proof-of-principle experiments on OMEGA and prospects for ignition on the National Ignition Facility", Phys. Plasmas 12056304 (2005)

[15] C. Stoeckl et al Phys. Rev. Lett. 90 235002-1 (2003)

[16] W. Seka et al. Phys. Plasmas 16052701 (2009) 